Published in final edited form as:

Crit Care Med. 2017 March ; 45(3): 556-557. doi:10.1097/CCM.0000000000002239.

\title{
Septic Cardiomyopathy: Getting to the Heart of the Matter
}

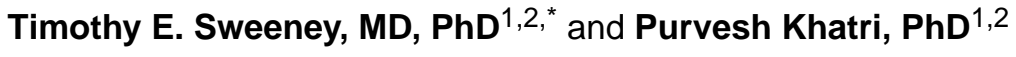 \\ ${ }^{1}$ Stanford Institute for Immunity, Transplantation and Infection, Stanford University School of \\ Medicine \\ ${ }^{2}$ Division of Biomedical Informatics Research, Department of Medicine, Stanford University \\ School of Medicine
}

\section{Keywords}

Keywords: Sepsis; cardiomyopathy; microarray; gene expression

\begin{abstract}
Sepsis is often accompanied by profound changes in the cardiovascular system, classically described as an initial hypodynamic state prior to resuscitation, followed by a hyperdynamic state with high cardiac output and low systemic vascular resistance. However, some patients also suffer from a reversible myocardial stunning known as 'septic cardiomyopathy', which manifests primarily as a depression in both right and left ventricular contractility (1). This septic cardiomyopathy is difficult to study since native physiological parameters are often augmented by clinical interventions such as fluid resuscitation and inotropes/vasopressors. Further, due to the obvious difficulty in sampling the heart directly, most studies on the underlying pathophysiology have focused on either circulating cytokines in a clinical setting, or on cellular or animal models (2). These prior studies have suggested that the dysregulated immune response in sepsis may be coupled to myocardial changes in nitric oxide production and signaling, mitochondrial function, and/or calcium-regulated contractility.
\end{abstract}

In this issue of Critical Care Medicine, Matkovich et al. report on a genome-wide expression profiling study of myocardium from 20 patients with sepsis immediately postmortem as compared to myocardium from 20 patients with other forms of cardiomyopathy, and to 11 non-failing donor hearts (3). This study represents an enormous amount of work by the clinical team, and an invaluable contribution from the patients and families who consented.

The clinical settings require close inspection to understand exactly what comparisons were made. The septic patients were mostly on pressors, indicating overall cardiovascular dysregulation. Transthoracic echocardiography was done in 12 of the 20 septic patients at a mean $5.6 \pm 2.5$ days prior to death. The patients showed a left ventricular ejection fraction of $53 \pm 5.1 \%$, though with a wide range (20-70\%). Coupled with the varying degrees of inotropic support, and the generally hyperdynamic state of post-resuscitation sepsis, it is hard to know what degree of septic cardiomyopathy existed in the studied population. This

\footnotetext{
*Corresponding author: tes17@ stanford.edu.

Competing Interests

Drs. Sweeney and Khatri are co-founders and stockholders of Inflammatix, Inc., which has a commercial interest in sepsis diagnosis.
} 
can be seen as both a strength of the study, in that a broad section of septic patients are represented (albeit only those that progressed to death), and a relative weakness, in that the findings are difficult to correlate with any particular clinical cardiomyopathic phenotype. We generally feel that the inclusion of a broader phenotype leads to results with more widespread applicability $(4,5)$.

The heart tissue from non-septic failing hearts was taken at time of explant, and heart tissue from non-failing hearts was taken from brain-dead organ donors. Notably, the non-septic cardiomyopathy group is split into both ischemic $(\mathrm{N}=11)$ and dilated $(\mathrm{N}=9)$ phenotypes, which are analyzed sometimes separately and sometimes jointly in the manuscript, based on the argument that prior studies have shown transcriptional differences between the two classes (6). Overall, the study allows for a reasonably informative comparison of the underlying transcriptomics between septic cardiomyopathy and other forms of cardiomyopathy.

Matkovich et al. first limited the genes under study to those which had been shown to be expressed at a reasonable level in a previous RNA-seq study of human hearts (6), reducing the number of included variables and increasing the likelihood of finding a biologically meaningful signal. They then showed using dimensionality-reduction techniques that the profiles of the septic hearts are overall substantially different from the other hearts profiled. A more in-depth analysis found that 169 genes were dysregulated in both septic and nonseptic failing hearts, including standard markers of cardiac stress (atrial natriuretic peptide and B-type natriuretic peptide). However, the authors found far more genes $(1,185)$ dysregulated in only the septic hearts, and focused the rest of the study on interpreting these genes.

The differences present in septic hearts but not non-septic failing hearts included pathways involved in metabolism and mitochondrial function, as well as pathways involved in sarcomere contraction and excitation/contraction coupling. The mitochondrial pathways were mostly downregulated (underexpressed) in the septic hearts as compared to the nonseptic failing hearts. Put in the context of prior studies, the dysregulation of mitochondrial genes can be seen as overall confirmatory. Other investigators have shown that the cytopathic hypoxia of sepsis leads to a reduced aerobic respiratory capacity (7), while prior transcriptomic profiling of skeletal muscle biopsies from patients with sepsis show both upregulated and downregulated mitochondrial genes (8). One question is whether the direction of change is generally representative of the patient population. Did these patients expire because they couldn't induce mitochondrial biogenesis (couldn't compensate), or is the downregulation of mitochondrial genes somehow an adaptive response? The authors' findings of dysregulation in sarcomeric and excitation/contraction coupling pathways similarly cannot be interpreted as either adaptive or maladaptive, but provide an interesting hypothesis for further study, as they suggest that intrinsic changes in the transcriptional machinery behind contractility may be altered in sepsis. More work will be needed to answer these questions, ideally by some sort of comparison between patients who survive sepsis and those who do not, as well as interventional studies in animal models. 
Overall, short of biopsies from still-beating hearts, the deeper question of what changes underlie septic cardiomyopathy at a cellular level remains unanswerable. However, Matkovich et al. have provided a valuable addition to the field. Gene expression profiles from patients who died of sepsis show differences in pathways related to both energy metabolism and cardiac contractility, suggesting that septic cardiomyopathy is both complex and separate from other cardiomyopathies.

\section{Acknowledgments}

Copyright form disclosure: Dr. Sweeney's institution received funding from Bill \& Melinda Gates Foundation, He received support for article research from the Bill \& Melinda Gates Foundation. Dr. Sweeney and Dr. Khatri disclosed receiving funding from owning stock in Inflammatix, Inc. Dr. Khatri received support for article research from National Institutes of Health and from the Bill \& Melinda Gates Foundation.

\section{References}

1. Vieillard-Baron A. Septic cardiomyopathy. Ann Intensive Care. 2011; 1(1):6. [PubMed: 21906334]

2. Flynn A, Chokkalingam Mani B, Mather PJ. Sepsis-induced cardiomyopathy: a review of pathophysiologic mechanisms. Heart Fail Rev. 2010; 15(6):605-611. [PubMed: 20571889]

3. Matkovich S, Al Khiami B, Efimov I, et al. Widespread Downregulation of Cardiac Mitochondrial and Sarcomeric Genes in Patients with Sepsis. Critical Care Medicine. 2017 IN PRESS.

4. Sweeney TE, Shidham A, Wong HR, et al. A comprehensive time-course-based multicohort analysis of sepsis and sterile inflammation reveals a robust diagnostic gene set. Sci Transl Med. 2015; 7(287):287ra271.

5. Sweeney TE, Haynes WA, Vallania F, et al. Methods to increase reproducibility in differential gene expression via meta-analysis. Nucleic Acids Res. 2016

6. Yang KC, Yamada KA, Patel AY, et al. Deep RNA sequencing reveals dynamic regulation of myocardial noncoding RNAs in failing human heart and remodeling with mechanical circulatory support. Circulation. 2014; 129(9):1009-1021. [PubMed: 24429688]

7. Levy RJ. Mitochondrial dysfunction, bioenergetic impairment, and metabolic down-regulation in sepsis. Shock. 2007; 28(1):24-28. [PubMed: 17483747]

8. Fredriksson K, Tjäder I, Keller P, et al. Dysregulation of mitochondrial dynamics and the muscle transcriptome in ICU patients suffering from sepsis induced multiple organ failure. PLoS One. 2008; 3(11):e3686. [PubMed: 18997871] 